\title{
AN OVERVIEW ON THE PREVALENCE OF DRUG RESISTANT STREPTOCOCCUS MUTANS IN DENTAL CARIES PATIENTS
}

\author{
Chowdaiah $\mathbf{M}^{1}$, Santosh Kumar ${ }^{2}$, Dhamodhar $\mathbf{P}^{3}$ \\ ${ }^{I}$ Department of Biotechnology, M. S. Ramaiah Institute of Technology, Bangalore-560054 \\ ${ }^{2}$ Department of Conservative Dentistry \& Endodontics, Faculty of Dental Sciences, M.S.Ramaiah University of \\ Applied Sciences, Bangalore - 560054 \\ ${ }^{3}$ Department of Biotechnology, M. S. Ramaiah Institute of Technology, Bangalore-560054
}

\begin{abstract}
Drug resistance is a worldwide problem. Drug resistant bacteria are more common in health clinic. Streptococcus mutans is the etiological agent of dental plaque. Over a number of decades epidemiological studies on human populations have indicated an association between the presence of Streptococcus mutans in plaque and their prevalence in dental caries. S. mutans has been implicated as cariogenic bacteria, because they produce high level of lactic acid and extracellular polysaccharide. It is observed that S.mutans is gaining resistance to commonly prescribed drugs. In the present study dental plaque samples were collected from patients of different locality within Bangalore city at M. S. Ramaiah Dental College, to study the pattern of drug resistance. Among 100 dental samples collected, 40 samples were grouped under Bangalore urban population and S. mutans was isolated. The isolates were identified as $S$. mutans by standard biochemical tests and fermentation studies. The isolates which showed positive results for gram staining and fermentation test were further confirmed with haemolysis test. The subculture of this S. mutans isolates were maintained in TH slants for further analysis.
\end{abstract}

Antibiotic Sensitivity test with commonly used antibiotics signified the emergence of drug resistance in S. mutans. Out of 40 isolates 23 isolates were drug-resistant S. mutans. About $56 \%$ isolates showed sensitivity to penicillin and Ciprofloxacin, while $30 \%$ of the isolates showed sensitivity to Erythromycin and $70 \%$ showed complete resistance. The present study indicated the increased resistance in $S$. mutans of urban population, towards the effective antibiotics Penicillin, Erythromycin and Ciprofloxacin.

Keywords: Antibiotics, CLSI guidelines, Multidrug resistance, Sensitivity, Dentalcaries

\section{INTRODUCTION}

Streptococcus mutans is a major causative agent for dental caries hence it is also called as cariogenic mutans streptococci(Houte, 1994). Over a few decades, extensive research carried out on cariogenic streptococci found that $S$. mutans is gradually acquiring resistance to commonly used antibiotics and are called as drug resistant $S$. mutans (Jubair, 2015; McGowan, 1983, 2006; Prakash et al., 2014). Drug resistance is of different types: multidrug-resistant (MDR), extensively drug-resistant (XDR) and pan drug-resistant (PDR) etc.

These drug resistant terms are being used in the medical literature to characterize the different patterns of resistance found in healthcare-associated, antimicrobial-resistant bacteria. If $S$. mutans is resistant to two or more antibiotics then it is called as multi-drug resistant $S$. mutans. These are more common in hospitals of developing countries where the antibiotics use is more(Dellit et al., 2007; McGowan, 1983).

Dental caries is a major health problem in school children and adults among these specifically sugar consuming people are most prone to get it. Countries like Canada, Egypt, Newzealand and etc have taken it as a major health concern and provided more percentage of their annual budget. With this we can pressume that dental caries should be treated more efficiently. Currently, mechanical removal of teeth with dental caries is adopted to control the spreading of cariogenic S. mutans(Anderson et al., 2006). But, it is not a good solution for initial and mid stages of dental caries instead can be controlled with antibiotics and proper maintenance of teeth.

There are various commercial drugs available for dental caries control and these are divided into 3 lines, first, second and third line drugs(Kaur et al., 2015). Recent studies on cariogenic $S$. mutans showed gradual increase in resistant pattern to antibiotics like, Penicillin, Erythromycin, Clindamycin, Ciprofloxacin, Metronidazole and Tetracycline(Prakash et al., 2014). Antimicrobial resistance is not new, but the number of resistant organisms, the geographic locations affected by drug resistance, and the breadth of resistance in single organisms are unprecedented and mounting(Levy \& Marshall, 2004). Based on the extensive research it is found that $S$. mutans gradually gaining resistance to commonly prescribed drugs worldwide due to continuous use. In this post antibiotic era, this problem of increasing antimicrobial resistance is even more threatening when considering the very limited number of new antimicrobial agents that are in development(Cars et al., 2005). 
Over a decade many researchers found that medicinal plant and their metabolites showing effective antibacterial patterns against drug-resistant bacteria. Plant materials like leaves, flowers, flower buds, root, bark, fruits, fruit peels and seeds are useful in controlling drug resistant $S$. mutans. Continuous research has been carrying out to find the active principle of phytochemicals which have medical implications. Natural herbs such as Tea, Neem, Tulsi, Cardamom (clove), Ginger, Nutmeg, Garlic and Pomegranate have been proved as effective in controlling the dental pathogens(Al-Daihan et al., 2013; Fani et al., 2007; González-Lamothe et al., 2009; Prakash et al., 2014; Sasidharan et al, 2011).

In present study, dental samples $(n=100)$ with caries were collected from patients over a period of March 2015-August 2015 visiting MS Ramaiah Dental college with prior consent, and samples from Bangalore urban population $(n=40)$ were grouped and sub-cultured. Multidrug resistant isolates were identified by biochemical test and drug resistance pattern was analyzed against Ciprofloxacin, Erythromycin and Penicillin $G$ as per the Clinical Laboratory Standards Institute (CLSI) guidelines(Table 1). Percentage of resistance was analyzed and compared with previously published data of Bangalore urban population and other major cities in India(Figure-2D). This study helps in knowing current resistance pattern and percentage of resistance among the Bangalore urban population.

\section{MATERIALS AND METHODS}

The Dental plaque samples were collected (from March 2015 to August 2015) at the M. S. Ramaiah Dental College in Todd Hewitt broth. They were transported to the M. S. Ramaiah Institute of Technology, Biotechnology Laboratory (temperature was controlled at $4^{\circ} \mathrm{C}$ ). There they were isolated, identified by simple tests like Gram staining (Collee, 1996), Phenol red (Cheesbrough, 2000) and Haemolysis (Buchanan et al., 1974; Slots et al., 1992) and sub-cultured for future use. Drug resistant patterns of samples were done by Kirby-Baver Disc-Diffusion method (Kirby et al., 1966). Standard lyophilized cultures were procured from the Microbial Type Culture Collection \& Gene Bank (MTCC), Chandigarh, namely Streptococcus mutans MTCC 497. This culture was revived using Todd Hewitt (TH) broth. Ciprofloxacin (05 $\mu \mathrm{g})$, Erythromycin (15 $\mu \mathrm{g})$, Penicillin G (10 units), Ampicillin (10units), Clindamycin (2units), Azithromycin (30units), Amoxycillin(10units), Gentamycin(10units) discs and Bacitracin were obtained from Hi-media. The results were recorded and it was determined whether the isolate was susceptible or resistant based on the CLSI guidelines.

\section{ANTIBIOTIC SUSCEPTIBILITY TESTING OF STREPTOCOCCUS MUTANS}

The Muller Hinton Agar plates were inoculated with the $S$. mutans sub-cultures along with the standard using separate sterile cotton swab for each bacterium. The swab immersed in the overnight culture tube, squeezed at the walls of tube to remove excess. The swab then taken and streaked on the surface of the MHA plate thrice, by rotating the plate $60^{\circ}$ after each streaking. Finally the swab was run around the edges of the agar to ensure the whole surface has been seeded. The culture was allowed to dry on the plate for 5 to 10 minutes at room temperature with the top in place. The plates were incubated for 24 hours at $37^{\circ} \mathrm{C}$ (aerobically). The plates were prepared in triplicates. The zones of inhibition to the nearest $\mathrm{mm}$ for each of sample was measured and the results were recorded. The zone size of the isolates and standard were compared and the pattern of drug resistance was analyzed.

\section{RESULTS AND DISCUSSION}

Dental caries due to streptococcal infection is most common and dangerous health problem in developing countries(Okeke et al., 2006). In India, major cities like Delhi, Bangalore (Fani et al., 2007; Prakash et al., 2014) and Tirupur (Karikalan et al., 2016) the research was done to find out the occurrences of multi-drug resistant $S$. mutans from dental samples. A couple of studies on Bangalore urban population from last 20 years showed the remarkable increase in prevalence of multi-drug resistant $S$. mutans.

In present study, initially screening and identification of $S$. mutans was done by biochemical tests like gram staining, phenol red test (Mannitol fermentation and Sorbitol fermentation) and $S$. mutans was isolated from 40 samples by using swab test. The incidence of $S$. mutans occurrence in dental samples was found to be $57.5 \%$ which is higher than the previous studies $(38 \%, 37 \%)$. S. mutans was confirmed by using blood agar haemolysis test in 23 isolates, and subjected for disc diffusion test (Kirby-Bauer et al,1966) along with standard MTCC culture and found different sensitivity or resistance patterns (Fig-1A). The sensitivity or resistance of $S$. mutans in isolates was determined by the zone of inhibition observed in disc diffusion test as per CLSI guidelines.(Table 1).

Table-1: CLSI limits for the antibiotic discs Penicillin G, Erythromycin, Ciprofloxacin, Ampicillin, Amoxacillin, Clindamaycin, Gentamycin and Azithromycin.

\begin{tabular}{|l|l|l|l|l|}
\hline Antibiotic & $\begin{array}{l}\text { Symb } \\
\text { ol }\end{array}$ & $\begin{array}{l}\text { Disc } \\
\text { Conten } \\
\mathbf{t}\end{array}$ & $\begin{array}{l}\text { Resista } \\
\mathbf{n t} \\
(\leq \mathbf{m m})\end{array}$ & $\begin{array}{l}\text { Sensitiv } \\
\mathbf{e}(\geq \mathbf{m m})\end{array}$ \\
\hline Penicillin G & PG & $\begin{array}{l}10 \\
\text { Units }\end{array}$ & 19 & 28 \\
\hline $\begin{array}{l}\text { Erythromyci } \\
\text { n }\end{array}$ & ER & $15 \mathrm{mcg}$ & 15 & 21 \\
\hline $\begin{array}{l}\text { Ciprofloxaci } \\
\text { n }\end{array}$ & RC & $5 \mathrm{mcg}$ & 15 & 21 \\
\hline Ampicillin & AM & $5 \mathrm{mcg}$ & 15 & 21 \\
\hline Amoxycillin & A & $30 \mathrm{mcg}$ & 15 & 22 \\
\hline Clindamycin & CD & $2 \mathrm{mcg}$ & 22 & 26 \\
\hline Gentamycin & G & $10 \mathrm{mcg}$ & 12 & 17 \\
\hline $\begin{array}{l}\text { Azithromyci } \\
\text { n }\end{array}$ & AT & $30 \mathrm{mcg}$ & 14 & 19 \\
\hline
\end{tabular}


The clinical isolates were subjected to antibiotic susceptibility pattern for the following drugs: Ciprofloxacin $(5 \mu \mathrm{g})$, Penicillin-G (10 units), Erythromycin $(15 \mu \mathrm{g})$, Ampicillin (10units), Clindamycin (2units), Azithromycin(30units), Amoxycillin(10units) and Gentamycin(10units). Among these Ciprofloxacin, Erythromycin and Penicillin G were selected, because they are effective and commonly used antibiotics against $S$. mutans (Prakash et al., 2014). Among the 23 isolates, 7 isolates were sensitive and 16 isolates were resistant to drugs used. Among the resistant isolates $6(20.7 \%)$ isolates showed resistant to Ciprofloxacin, $15(51.7 \%)$ isolates to Erythromycin and $8(27.6 \%)$ isolates showed resistance to all antibiotics used (Fig-1D).

In a previous study from Bangalore urban population showed that $38 \%$ high rate of incidence of S. mutans (Prakash et al., 2014). Recently in a similar study at Tirupur, India revealed that cariogenic S. mutans was $14 \%$ resistant to Erythromycin and $100 \%$ resistant to Penicillin-G. Similarly when we compare the same with worldwide these values are significantly more (Karikalan et al., 2016). The incidence of MDR in Iran was found to be $30.4 \%$ (Fani et al., 2007). In Egypt, 45 isolates showed 32\%, 11\% \& $8 \%$ resistance to Ciprofloxacin, Erythromycin \& Penicillin-G respectively in the Cairo, Egypt population (El Sherbiny, 2014).
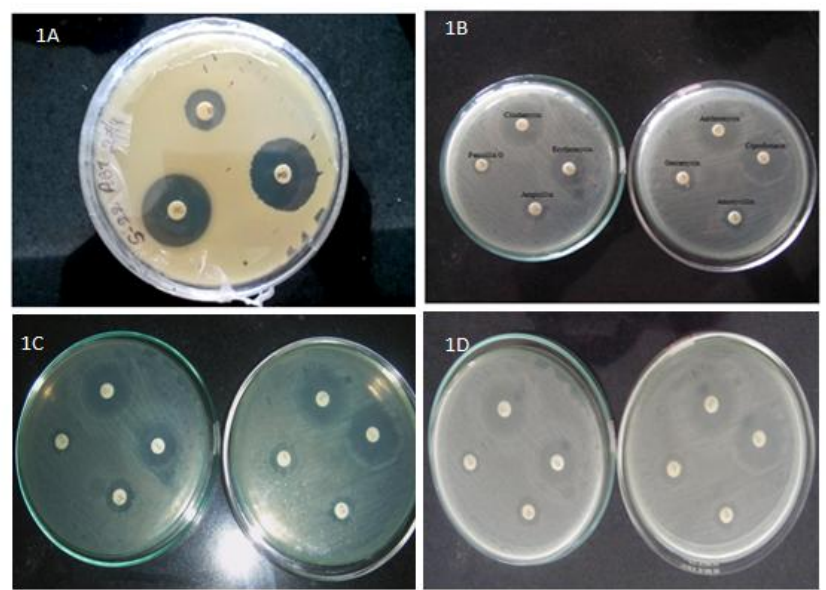

Fig-1; A. Anti-biogram of Standard MTCC culture for Erythromycin (ER), Ciprofloxacin (RC) \& Penicillin G (PG), 1B, 1C \& 1D were multidrug resistant antibiograms of isolates against Clindamycin, Penicillin G, Erythromycin, Ampicillin, Azithromycin, Gentamycin, Amoxycillin and Ciprofloxacin.

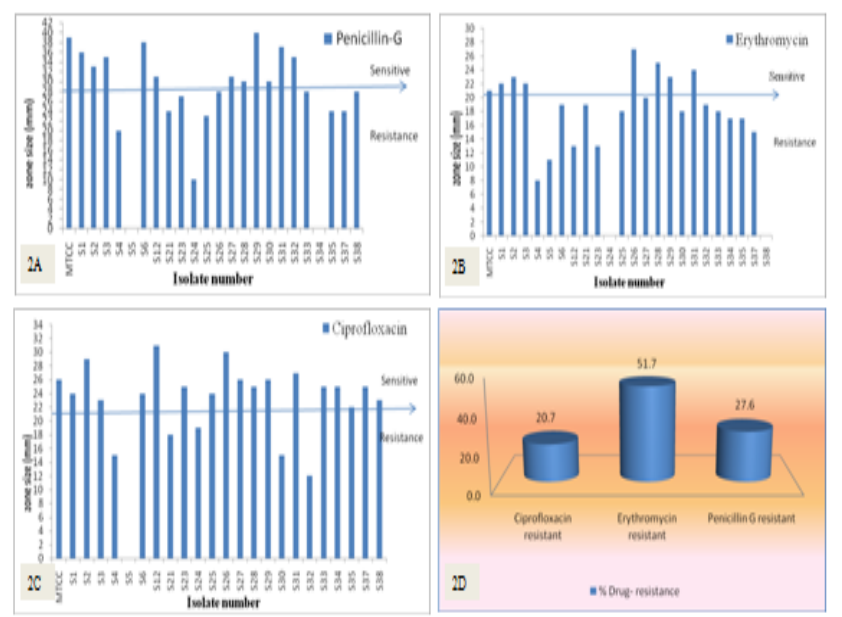

Fig-2A-Isolates showing antibiotic pattern for Penicillin G,

2B-Isolates showing antibiotic pattern for Erythromycin,

2C-Isolates showing antibiotic pattern for Ciprofloxacin and

2D- Percentage resistance of isolates against antibiotics Ciprofloxacin, Erythromycin and Penicillin G.

Even though, few drugs are still effective against drug resistant $S$. mutans adverse reactions of systemic drugs during last five years (2010-2014) for various following organ groups received: skin, gastrointestinal disorders, hepato-biliary disorders, general disorders, blood disorders, neurological reactions, respiratory disorders, immune system disorders, musculo-skeletal disorders, psychiatric disorders, renal and urinary disorders (Weinstein, 2001).

Various recent studies showed that medicinal plants and spices (Clove, Neem oil, Babool, Ginger, Nutmeg, Garlic and pomegranate, etc) are effectively used against these resistant $S$. mutans with minimum side effects(Al-Daihan et al., 2013; Fani et al., 2007; Prakash et al., 2014). The present study revealed that there is increased multidrug resistance in Bangalore urban population. The isolates showed increase in drug resistance to most commonly used antibiotics such as Erythromycin, Ciprofloxacin and Penicillin G.

\section{ETHICAL CONSIDERATIONS}

Permission to carry out this study was obtained from Ethical committee of MS Ramaiah Dental College. Written consent was sought and obtained.

\section{CONCLUSION}

Based on the above studies it was found that urban population in Bangalore, India showed increased drug resistant Streptococcus mutans. Over a period of five years there is tremendous increase in resistance for commercially available antibiotics like Penicillin G, Ciprofloxacin and Erythromycin. There is a need of pure natural antibiotics which can act against MDR bacteria with no severe side effects to human. 


\section{REFERENCES}

[1] Al-Daihan, S., Al-Faham, M., Al-shawi, N., Almayman, R., Brnawi, A., shafi Bhat, R., \& others. (2013). Antibacterial activity and phytochemical screening of some medicinal plants commonly used in Saudi Arabia against selected pathogenic microorganisms. Journal of King Saud UniversityScience, 25(2), 115-120.

[2] Anderson, M. H., \& Shi, W. (2006). A Probiotic Approach to Caries Management. Pediatric Dentistry, 28(2), 151-153.

[3] Buchanan, R. E., \& Gibbons, N. E. (1974). Bergey's Manual of Determinative Bacteriology, eight edition. Board. The Williams and Wilkins, Baltimore.

[4] Cars, O., \& Nordberg, P. (2005). Antibiotic resistance-The faceless threat. International journal of risk and safety in medicine, 17(3), 103-110.

[5] Cheesbrough, M. (2000). District Laboratory Practice in Tropical Country. Part 2. Cambridge university press.

[6] Collee, J. G. (1996). Mackie \& Mccartney Practical Medical Microbiology (14Th Edition). Elsevier (A Divisionof Reed Elsevier India Pvt. Limited).

[7] Dellit, T. H., Owens, R. C., McGowan, J. E., Gerding, D. N., Weinstein, R. A., Burke, J. P., ... others. (2007). Infectious Diseases Society of America and the Society for Healthcare Epidemiology of America guidelines for developing an institutional program to enhance antimicrobial stewardship. Clinical Infectious Diseases, 44(2), 159-177.

[8] El Sherbiny, G. M. (2014). Control of growth Streptococcus mutans isolated from saliva and dental caries. Int. J. Curr. Microbiol. App. Sci, 3(10), 1-10.

[9] Fani, M. M., Kohanteb, J., Dayaghi, M., \& others. (2007). Inhibitory activity of garlic (Allium sativum) extract on multidrug-resistant Streptococcus mutans. Journal of Indian Society of Pedodontics and Preventive Dentistry, 25(4), 164.

[10] González-Lamothe, R., Mitchell, G., Gattuso, M., Diarra, M. S., Malouin, F., \& Bouarab, K. (2009). Plant antimicrobial agents and their effects on plant and human pathogens. International Journal of Molecular Sciences, 10(8), 3400-3419.

[11] Houte, J. van. (1994). Role of Micro-organisms in Caries Etiology. Journal of Dental Research, 73(3), 672-681.

[12] Jubair, H. H. (2015). The Relationship Between Biofilm Forming and Antibiotics Resistance of Streptococcus mutans Isolated From Dental Caries. Int. J. Curr. Microbiol. App. Sci, 4(5), 568-574.

[13] Karikalan, S., \& Mohankumar, A. (2016). Studies on ampicillin resistant plasmid of Streptococcus mutans isolated from dental caries patients.

[14] Kaur, N., Sahni, P., Singhvi, A., Hans, M. K., \& Ahluwalia, A. S. (2015). Screening the Drug Resistance Property Among Aerobic Pathogenic Microorganisms of Dental Caries in North-Western Indian Population: A Preliminary Study. Journal of Clinical and Diagnostic Research: JCDR, 9(7), ZC05-ZC08.
[15] Kirby MDK, Bauer RW ,Sherris J C,Turck M(1966) Antibiotic susceptibility testing by standard disc diffusion method. Am J Clin Pathol 45:493-496.

[16] Levy, S. B., \& Marshall, B. (2004). Antibacterial resistance worldwide: causes, challenges and responses. Nature Medicine, 10, S122-S129.

[17] McGowan, J. E. (1983). Antimicrobial resistance in hospital organisms and its relation to antibiotic use. Review of Infectious Diseases, 5(6), 1033-1048.

[18] McGwan, J. E. (2006). Resistance in nonfermenting gram-negative bacteria: multidrug resistance to the maximum. The American Journal of Medicine, 119(6), S29-S36.

[19] Okeke, I. N., Laxminarayan, R., Bhutta, Z. A., Duse, A. G., Jenkins, P., O'Brien, T. F., ... \& Klugman, K. P. (2005). Antimicrobial resistance in developing countries. Part I: recent trends and current status. The Lancet infectious diseases, 5(8), 481-493.

[20] Prakash, D., Ramesh, K., Gopinath, N., Varuvelil, G. J., \& others. (2014). Antibacterial efficacy of Syzygium aromaticum extracts on multi-drug resistant Streptococcus mutans isolated from dental plaque samples. Journal of Biochemical Technology, 3(5), 155-157.

[21] Sasidharan, S., Chen, Y., Saravanan, D., Sundram, K. M., \& Latha, L. Y. (2011). Extraction, isolation and characterization of bioactive compounds from plants' extracts. African Journal of Traditional, Complementary and Alternative Medicines, 8(1).

[22] Slots, J., \& Taubman, M. A. (1992). Contemporary oral microbiology and immunology. Mosby Year Book.

[23] Weinstein, R. A. (2001). Controlling antimicrobial resistance in hospitals: infection control and use of antibiotics. Emerging Infectious Diseases, 7(2), 188192. 